\title{
L'enseignement du français avec les interactions : approche méthodologique et mise en œuvre en classe depuis le niveau débutant
}

\author{
Carole ETIENNE ${ }^{1}$ et Catherine DAVID ${ }^{2}$ \\ ${ }^{1}$ CNRS Laboratoire Interactions Corpus Apprentissages Représentations (ICAR, UMR5191) - ENS \\ Lettres, 15 parvis Descartes, 69007 Lyon \\ ${ }^{2}$ Laboratoire Parole et Langage (LPL, UMR 7309), 5 avenue Pasteur, 13100 Aix-en-Provence
}

\begin{abstract}
Résumé. Si les enseignants considèrent la compétence d'interaction dans les situations professionnelles ou privées du quotidien comme fondamentale dans l'acquisition d'une langue, son enseignement reste encore problématique pour intégrer la diversité des situations et préparer l'apprenant à acquérir certains automatismes qui lui permettront de mieux les comprendre et d'intervenir plus facilement. Pour tenter de pallier ce manque, les linguistes spécialistes des interactions ont décidé de constituer des ressources en partenariat avec les praticiens impliqués dans des enseignements de Français Langue Etrangère (FLE) à destination de publics variés. Cet article entend présenter l'approche méthodologique qui a permis d'articuler les travaux des chercheurs en linguistique avec les besoins identifiés par les didacticiens et la mise en œuvre de ces nouveaux supports multimédia en situation de classe, notamment pour un public débutant pour lequel les interactions naturelles semblent à priori difficiles à prendre en main.
\end{abstract}

\begin{abstract}
Teaching French as a Foreign Language with interactions : methodological approach and resources usages in class situation, in particular for a beginner public. If teachers consider the interactional skill in everyday professional or private situations as essential in language acquisition, teaching the diversity of social situations and preparing the learner to get certain automatisms in order to understand them and to interact easily remain difficult. Then, the linguists specialised in interaction have decided to build resources in partnership with practitioners involved in teaching French as a Foreign Language (FFL) available for different needs. This article aims to present the methodological approach that has made it possible to articulate the work of linguistic researchers with the needs identified by the didactic researchers. It gives some examples of resources usages in class situation, in particular for a beginner public where naturally occurring corpora are often considered not suitable and difficult to handle.
\end{abstract}

\section{L'étude des interactions}

Si l'étude des interactions ne peut bien entendu pas se résumer à un article ou à un paragraphe, nous allons rappeler quelques-uns de ces principes pour apporter un éclairage 
indispensable à la compréhension des enjeux et des difficultés que ces interactions posent aux apprenants, quel que soit leur niveau de langue.

Par rapport à d'autres expressions écrites ou orales d'une langue, l'interaction se caractérise avant tout par sa temporalité puisque le locuteur intervient dans un déroulement séquentiel sans avoir la possibilité d'en interrompre le cours pour se donner le temps nécessaire à la réflexion, effacer ou modifier ses propos ou ses gestes dans la mesure où ses interlocuteurs ont déjà réceptionné ce qu'il a produit dans un processus continu et dynamique. Tout au long de l'interaction, le locuteur qui est en train de s'exprimer va devoir également intégrer les interventions des autres locuteurs qui vont interrompre, compléter ou questionner ses propos sans attendre nécessairement que son développement soit terminé. Par conséquent, il ne pourra pas toujours réaliser les mêmes tâches de la même manière mais devra ajuster ses propos aux réactions des autres locuteurs pour pouvoir y répondre et mener à bien son échange (Sacks, Schegloff \& Jefferson, 1974).

Par ailleurs, le locuteur sera amené à prendre en compte d'autres modalités non verbales qu'il devra là encore interpréter in situ comme la prosodie, les regards, les gestes, les postures (Goodwin 2000) ou la manipulation d'artefacts qui vont modifier, ou même parfois remplacer, les productions verbales, comme c'est le cas pour une intonation montante qui transforme un énoncé en question, un hochement de tête ou un geste de pointage sur un document pour apporter une réponse (Mondada, 2006, 2007) ou bien un regard appuyé pour solliciter un locuteur. Enfin, il devra s'adapter au contexte dans lequel l'interaction se déroule, qui va parfois l'amener à réaliser différemment la même fonction, selon Kerbrat Orecchioni (2001)

« [...] la connaissance des formules pour exprimer cet acte de langage est tout à fait nécessaire, mais elle n'est pas suffisante : il faut également savoir dans quelles circonstances il convient de l'employer et dans quels cas il est préférable de ne pas en user $»$.

De plus, le locuteur devra prendre conscience de certaines caractéristiques interculturelles qui peuvent modifier sa perception, son interprétation et par suite sa compréhension, lui évitant ainsi des réponses inadaptées à la culture dans la situation en cours pouvant mettre en jeu les finalités de l'échange (Kerbrat-Orecchioni 2001, 2002).

En parallèle de ces caractéristiques générales, les chercheurs se sont intéressés plus spécifiquement à la description de l'organisation de ces interactions (Traverso, 2016) avec la structuration des tours de parole, leur alternance, la co-construction des actions entre les participants et l'ensemble des ressources verbales et multimodales mobilisées par les interactants pour articuler les activités interactionnelles afin de mener à bien leur échange.

Alors que les travaux fondateurs de Kerbrat-Orecchioni (1990-1994) ont initié et développé ce champ de recherche pour la langue française, certaines situations d'interaction ont fait l'objet de recherches dédiées comme les conversations familières (Traverso, 1998), les interactions dans les commerces (Kerbrat-Orecchioni/Traverso, 2008), les réunions professionnelles (Détienne \& Traverso, 2009), la conversation en ligne (Traverso, 2011), les visites guidées (Traverso, 2017) ou plus récemment l'accueil des migrants (Piccoli, Ticca \& Traverso, 2019).

Au-delà des variations situationnelles que nous venons d'exposer, les chercheurs ont dégagé des récurrences qu'ils ont définies comme des "méthodes " que les locuteurs mettent en œuvre au cours de l'interaction pour atteindre leurs objectifs (Sacks, 1992) et qui donnent aux interactions une structuration qui garantit leur bon déroulement et leur finalité : réalisation d'un achat, prise de décision en réunion, changement de sujet, préparation d'un plat, invitation, repas, compréhension des explications d'un médecin, explication d'un 
itinéraire, prise d'un rendez-vous, compréhension des règles d'un jeu, visite d'un monument...

Pour y parvenir, les linguistes s'appuient sur l'analyse d'interactions naturelles ou écologiques dans le sens où elles sont filmées ou enregistrées à l'endroit même où elles se déroulent, sans intervention, ni recommandation, ni consigne donnée par le chercheur aux locuteurs (Potter, 2006). Il s'agit de situations ordinaires du quotidien dans un commerce, en réunion professionnelle, dans un repas en famille ou entre amis, lors d'une consultation médicale, un jeu, une visite guidée ou plus généralement une conversation que ce soit en face à face ou bien à distance, au téléphone ou en visioconférence. Un ensemble de métadonnées sur le contexte, les locuteurs et l'enregistrement est recueilli et sera utilisé au cours des analyses détaillées ou pour créer ultérieurement des collections. Un travail minutieux de transcription est réalisé manuellement pour annoter les productions verbales et non verbales (intonation, enchaînement rapide, chevauchement de parole, pause, chuchotement, saillance, allongement de syllabes...).

Les corpus oraux d'interaction ainsi constitués par les chercheurs sont rendus disponibles à la communauté dans la banque de données CLAPI (http://clapi.icar.cnrs.fr) où ils peuvent être téléchargés, requêtés pour constituer des collections multimédia ou bien pour disposer d'analyses quantitatives sur les fréquences, les co-occurrences ou les contextes d'emploi (Groupe ICOR, 2016). À ce jour, 63h de données sont accessibles mais l'alimentation de la base se poursuit en fonction des nouveaux projets de recherche.

Les ressources que nous allons présenter intègrent la diversité de ces situations et les résultats de certains de ces travaux de recherche afin de donner la possibilité à l'enseignant d'exposer l'apprenant à cette variété mais aussi d'en expliquer les mécanismes pour qu'il puisse les interpréter en situation (in situ), s'approprier leur sens et leur structure.

\section{La compétence d'interaction décrite dans le CECRL}

Comme nous venons de le voir, il est difficile de parler d'une seule compétence alors que les procédés mis en œuvre dans l'interaction font appel à un ensemble de compétences de la part des participants dans un processus continu dynamique où ils coordonnent leurs productions pour co-construire cet échange et parvenir à sa finalité.

Du point de vue de l'apprenant, la compétence d'interaction concerne à la fois la réception donc la compréhension des attentes des autres locuteurs et l'émission c'est à dire la production pour initier ou répondre aux sollicitations, sachant comme nous venons de le voir qu'il s'agit de prendre en compte in situ le lexique et la syntaxe mais aussi d'autres dimensions : la prosodie, les gestes, les regards, les postures, les mouvements, ...

Cette compétence est décrite dans le Cadre Européen Commun de Référence pour les Langues (CECRL) en ces termes:

« Dans l'interaction, au moins deux acteurs participent à un échange oral et/ou écrit et alternent les moments de production et de réception qui peuvent même se chevaucher dans les échanges oraux. Non seulement deux interlocuteurs sont en mesure de se parler mais ils peuvent simultanément s'écouter. Même lorsque les tours de parole sont strictement respectés, l'auditeur est généralement en train d'anticiper sur la suite du message et de préparer une réponse. Ainsi, apprendre à interagir suppose plus que d'apprendre à recevoir et à produire des énoncés. On accorde généralement une grande importance à l'interaction dans l'usage et l'apprentissage de la langue étant donné le rôle central qu'elle joue dans la communication. » 
Si le CECRL accorde une place importante à l'interaction dès sa première édition qu'il développe largement dans le Complément (2018 : 86-89), il n'en demeure pas moins qu'il appréhende la compréhension des échanges en interaction seulement à partir du niveau B2. Même si les apprenants B1 peuvent « aborder sans préparation une conversation sur un sujet familier » (CECRL, 2018 : 88), le CECRL mentionne l'importance d'un langage « clairement articulé » où on « devra quelquefois faire répéter certains mots ou expressions » tandis que les apprenants de niveau B2 pourront comprendre «ce qu'on leur dit en langue standard» (CECRL, 2018 : 87). Ce n'est qu'à partir du niveau C1 qu'on estime que l'apprenant « peut utiliser la langue en société avec souplesse et efficacité, y compris dans un registre affectif, allusif ou humoristique » (CECRL, 2018 : 88). L'acquisition d'une compétence réelle d'interaction est seulement attribuée au niveau $\mathrm{C} 2$ où l'apprenant «peut converser de façon confortable et appropriée sans qu'aucune limite linguistique ne vienne empêcher la conduite d'une vie personnelle et sociale accomplie » (CECRL, 2018 : 88).

Il semblerait donc difficile d'introduire les interactions authentiques des corpus oraux de CLAPI en situation de classe avant le niveau B2.

\section{COMPRENDRE UN INTERLOCUTEUR}

PROSIGN

Peut comprendre tout interlocuteur même sur des sujets spécialisés abstraits ou complexes et hors de son domaine, à condition d'avoir l'occasion de s'habituer à un accent moins familier.

Peut comprendre en détail une intervention sur des sujets spécialisés abstraits ou complexes même hors de son domaine, mais peut avoir besoin de faire confirmer quelques détails, notamment si l'accent n'est pas familier.

B2 Peut comprendre en détail ce qu'on lui dit en langue standard mème dans un environnement bruyant.

B1 Peut suivre un discours clairement articulè et qui lui est destiné dans une conversation courante, mais devra quelquefois faire répéter certains mots ou expressions.

\section{Peut comprendre suffisamment pour gérer un échange simple et courant sans effort excessif.}

Peut généralement comprendre un discours clair et standard qui lui est adressé sur un sujet familier, à condition de pouvoir demander de répéter ou reformuler de temps à autre.

Peut comprendre ce qui lui est dit clairement, lentement et directement dans une conversation quotidienne simple, à condition que l'interlocuteur prenne la peine de l'aider à comprendre.

Peut comprendre des expressions quotidiennes pour satisfaire des besoins simples de type concret si elles sont répétées, formulées directement, lentement et clairement par un interlocuteur compréhensif.

Peut comprendre des questions et des instructions qui lui sont adressées lentement et avec soin et suivre des consignes simples et brèves.

Peut comprendre des questions simples qui le/la concernent directement, par exemple sur son nom, son âge et son adresse, ou d'autres choses de ce type, si on linterroge lentement et clairement.

Peut comprendre une information personnelle simple (par exemple le nom, l'âge, l'adresse, l'origine) quand quelqu'un se

Pré-A1 présente, à condition qu'il/elle lui parle directement, lentement et clairement, et peut comprendre des questions qui lui sont adressées sur ces mêmes thèmes, bien qu'elles doivent parfois être répétées.

Peut comprendre certains mots familiers et certaines salutations et reconnaitre une information importante telle que des chiffres, des prix, des dates, les jours de la semaine, à condition qu'on les lui dise et qu'on les répète si nécessaire, très lentement.

Fig. 1. CECRL 2018 Comprendre un interlocuteur, p 87 
Peut converser de facon confortable et appropriée sans qu'aucune limite linquistique ne vienne empécher la conduite d'une vie personnelle et sociale accomplie.

Peut établir une relation avec ses interlocuteurs, par des questions engageantes et en utilisant des expressions montrant quillille est d'accord ainsi que, si c'est le cas, en parlant d'une tierce personne ou de la situation commune.

Peut émettre des réserves et des réticences, fixer ses conditions quand ivelle accepte des demandes ou donne des autorisations, et demander si les gens ont compris sa position.

Peut s'impliquer dans une conversation d'une certaine longueur sur la plupart des sujets dintérét général en y participant réellement et ce mème dans un environnement bruyant.

Peut maintenir des relafions avec des locuteurs de la langue cible sans les amuser ou les irriter involontairement ou les obliger à se comporter autrement qu'ils ne le feraient avec tout autre locuteur.

Peut transmettre différents degrés d'émotions et souligner ce qui est important pour luilelle dans un événement ou une expérience.

Peut démarrer une conversation sur des sujets familiers et aider à la poursuivre en posant des questions assez spontanées sur une expérience ou un événement particulier, et exprimer ses réactions et son opinion.

Peut soutenir des conversations relativement longues sur des sujets dintérêt général, à condition que finterlocuteur fasse un effort pour faciliter la compréhension.

\section{Peut aborder sans préparation une conversation sur un sujet familier.}

Peut suivre une conversation quotidienne si linterlocuteur s'exprime clairement, bien qu'il lui soit parfois nécessaire de faire répéter certains mots ou expressions.

Peut soutenir une conversation ou une discussion mais risque d'être quelquefois difficile à suivre lorsquillielle essaie de formuler exactement ce quilitelle aimerait dire.

Peut réagir à des sentments tels que la surprise, la joie, la tristesse, la curiosité et rindifférence et peut les exprimer.

Peut établir un contact social : salutations et congé ; présentafions ; remerciements.

Peut généralement comprendre un discours standard clair, qui lui est adressé, sur un sujet familier, à condilion de pouvoir faire répéter ou reformuler de temps à autre.

Peut participer à de courtes conversations dans des contextes habituels sur des sujets généraux.

Peut dire en termes simples comment illelle va et remercier.

Peut demander à quelqu'un de lui rendre un service (par ex. de lui prêter quelque chose), et peut proposer de rendre un service et répondre si quelqu'un lui demande un service.

Fig. 2. CECRL 2018 Conversation, p 88

\section{L'approche méthodologique}

Les chercheurs en interaction du laboratoire ICAR se sont intéressés à la constitution de ressources adaptées à l'enseignement du français à partir de leurs travaux de recherche et de leurs corpus oraux qui concernent des interactions comme nous les vivons tous au quotidien et auxquelles un locuteur non natif serait confronté à son arrivée dans un pays francophone. Dans cette perspective, ils ont constitué et animé un réseau de didacticiens et d'enseignants en France et dans les départements de Français à l'étranger pour décider ensemble des étapes à suivre et élaborer au fur et à mesure des ressources que les praticiens pourraient éprouver en situation de classe, avec différents niveaux d'apprenants et pour différents objectifs.

Dans une première étape, les didacticiens ont proposé de vérifier que les interactions naturelles pourraient être comprises par des apprenants de différents niveaux de langue car la communauté enseignante émettait des réserves quant à ce matériel, l'évaluant comme particulièrement complexe compte-tenu des chevauchements de parole, du débit, des intonations et des bruits ambiants comparativement aux données des manuels où les locuteurs parlent les uns après les autres, avec un débit adapté au niveau de langue, dans un environnement non bruité. Un projet exploratoire CLAPI-FPIE a été réalisé avec les didacticiens du département français de Lund en Suède Anita Thomas, Jonas Granfeldt, 
Nina Bengtsson et Céline Rocher-Hahlin et Isabelle Guinamard didacticienne à l'ISPEF de l'Université Lyon 2 qui ont conçu un protocole où 4 extraits de désaccord ont été projetés à 9 étudiants suédois dont 3 de niveau A2/B1, 3 de niveau B2 et 3 de niveau $\mathrm{C} 1$, évalués par le même test de langue DIALANG. Après une brève contextualisation et un double visionnage, l'étudiant devait répondre oralement aux questions «Qu'est-ce qu'il se passe dans cet extrait ? De quoi parlent-ils ? » puis il leur était demandé après une troisième projection, d'identifier où commençait et se terminait le désaccord, c'est-à-dire la séquence en tant que telle et de justifier ce qui leur permettait de la déterminer. Les résultats de ce protocole (Thomas \& al, 2016) ont établi que si les apprenants les plus avancés étaient bien entendu plus à l'aise et comprenaient ces échanges de manière assez fine, les débutants se sont révélés capables de comprendre l'essentiel de l'échange de manière globale, leurs difficultés lexicales et syntaxiques les amenaient à prêter plus d'attention aux répétitions et aux autres dimensions : la prosodie, les rires, les regards, les expressions du visage et les gestes. En outre, les bruits ambiants jugés pénalisants leur apportaient au contraire des informations sur le contexte.

En parallèle, Natacha Niemants, didacticienne à l'Université de Bologne en Italie, a soumis les mêmes extraits à des étudiants futurs interprètes, donc d'un niveau de langue avancé, et a constaté que ces élèves étaient par contre perturbés en compréhension par les tours de parole courts à faible valeur lexicale qui se révèlent très fréquents en interaction, mais dont ils comprenaient souvent mal la finalité. Ce constat nous a conduit à développer dans nos ressources un focus sur les marqueurs de l'oral et sur les procédés utilisés par les locuteurs pour co-construire le sens au cours de l'échange.

Dans ces deux expérimentations, la nécessité d'expliquer le contexte avant le visionnage a été relevée.

\subsection{La sélection d'extraits adaptés à une session de cours}

Forts de cette première expérience validant l'utilisation de telles interactions authentiques même auprès d'élèves débutants (Holec, 1990), les chercheurs et les didacticiens ont décidé de concevoir ensemble une application en ligne rendant disponibles ces données pour l'enseignement, ils se sont orientés dans un premier temps vers des extraits d'interactions (Etienne \& Jouin, 2019). Les didacticiens ont suggéré des passages assez courts (autour de 2 minutes) pour être exploitables dans la durée d'un cours, contextualisés pour les introduire aux étudiants, didactisés en attribuant des thèmes et des objectifs et en suggérant des pistes d'exploitation ainsi qu'en identifiant les difficultés qu'ils pouvaient présenter qu'elles soient lexicales, syntaxiques, interculturelles ou liées au débit des locuteurs (Figure 3). Un niveau de langue au sens du CECRL, ou plus souvent une plage de niveau, a été associée pour s'adapter aux pratiques des enseignants, sachant que le même extrait peut bien entendu être étudié à différents niveaux selon l'exploitation demandée. Les linguistes ont ajouté les transcriptions de ces extraits en suggérant certains phénomènes par défaut (début de chevauchement de parole noté "[", pauses dont la durée figure entre parenthèses, descriptions, intonations représentées visuellement par des flèches montantes ou descendantes), tout en proposant à l'enseignant un affichage modulaire des phénomènes de l'oral afin de générer la transcription la plus appropriée à ses objectifs. En parallèle, un dictionnaire en ligne plus spécialement à l'attention des apprenants est disponible, ainsi qu'un relevé des mots les moins fréquents nécessitant probablement une explication de l'enseignant, complété par des informations quantitatives sur le débit des locuteurs et la variété lexicale. Les extraits de la même situation ont été indiqués si l'enseignant souhaite proposer un travail personnel complémentaire sur des données déjà étudiées en cours. 
Une quarantaine d'extraits des interactions de CLAPI a été ainsi mis en ligne dans la plateforme CLAPI-FLE (http://clapi.icar.cnrs.fr/FLE) dans le cadre d'une collaboration avec Florence Mourlhon-Dallies, professeure à l'Université Paris 5, par son étudiante Martina Ronci en concertation avec les interactionnistes.
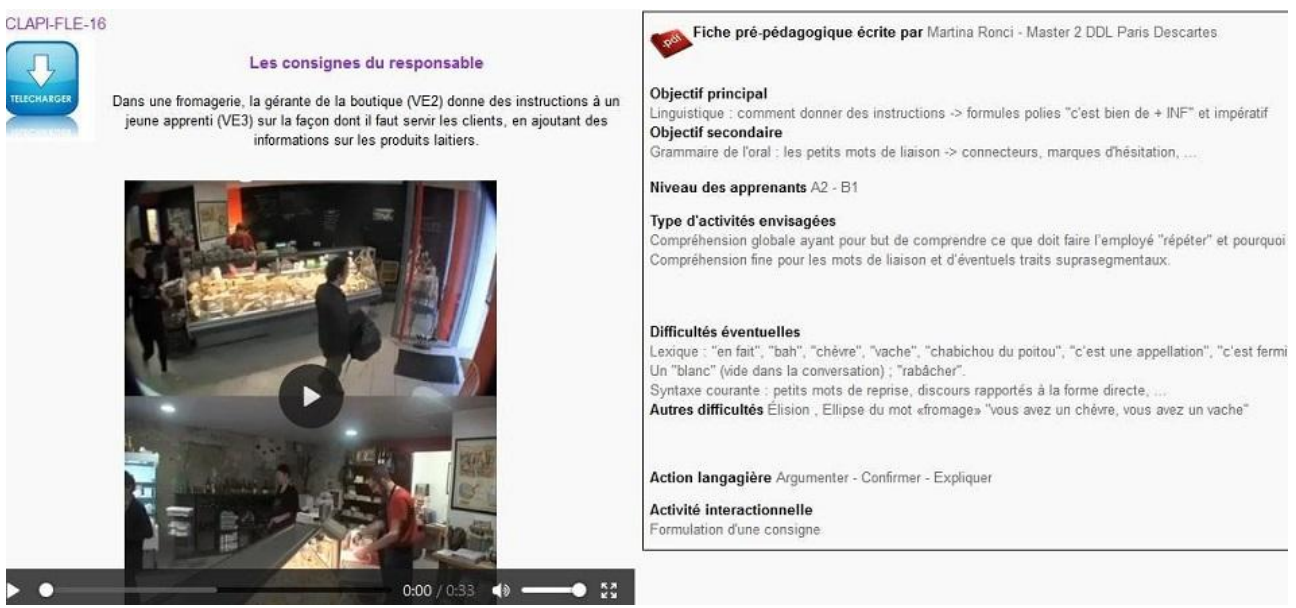

Fig. 3. Extrait CLAPI-FLE-23, onglet Exploitation

Ces extraits ont été utilisés en situation de classe auprès de différents publics et pour différents objectifs d'enseignement, on peut citer notamment Carmen Alberdi à l'Université de Grenade (Alberdi \& al, 2018) qui a fait appel à ces supports dans des enseignements dans des filières à vocation professionnelle en économie, en tourisme et dans des écoles de traduction, elle télécharge également directement les corpus de la banque de données CLAPI dont elle choisit des passages pour couvrir certains de ces besoins. Un autre usage concerne l'enseignement de la linguistique française avec l'exploitation réalisée par Elisa Ravazzolo à l'Université de Trento en Italie qui fait appel aux extraits de CLAPI-FLE pour sensibiliser les apprenants de niveau A2/B1 à l'identification et à l'analyse de phénomènes linguistiques saillants comme les assimilations, les élisions, les simplification de groupes consonantiques mais également à certaines structures syntaxiques (les clivées, les dislocations ...) et aux routines interactionnelles dans les échanges (Ravazzolo \& al, 2015). Nous détaillerons au chapitre 5 la mise en perspective d'une invitation dans une interaction construite proposée dans les manuels avec une interaction d'invitation téléphonique de CLAPI-FLE pour un public débutant.

\subsection{Les collections pour illustrer un fait de langue}

En parallèle de ces extraits, des collections ont été constituées pour illustrer certains phénomènes fréquents de l'oral comme les dislocations, l'imparfait de politesse, certaines structures "le truc c'est que", "c'est vrai que", "tu/vous suivi de l'indicatif". Leur format comprend une courte présentation, une typologie et quelques exemples pour chacune des catégories, incluant pour chacun d'eux une brève contextualisation, la vidéo ou l'audio en streaming ou en téléchargement et la transcription correspondante. D'un point de vue méthodologique, elles ont été réalisées par Sabine Hervé, étudiante en master didactique à l'Université Paris 5, puis relues et enrichies par Élodie Oursel, chercheuse en didactique à l'Université Paris 8. 
Après avoir étudié un extrait dans une situation d'interaction donnée où l'enseignant a relevé un procédé comme une dislocation ou l'usage de l'imparfait de politesse, il peut alors illustrer ce procédé dans des contextes différents ou bien proposer aux étudiants de reconnaître ce procédé et de l'expliquer dans les exemples donnés dans les collections.

\subsection{Les fiches pour expliquer de manière détaillée un fait de langue}

Dans un second temps, notre consortium de didacticiens et de linguistes a étudié de quelle manière les résultats des travaux de recherche en interaction pourraient être transposés à des fins pédagogiques pour répondre à des problématiques d'enseignement, en expliquant de manière détaillée certains faits de langue et en intégrant leurs différentes dimensions : lexicales avec en parallèle une rubrique consacrée à l'utilisation des marqueurs de l'oral à la fois fréquents et parfois problématiques pour les apprenants qui ont des difficultés à leur attribuer un sens, syntaxiques, prosodiques, interculturelles et multimodales (regards, gestes, mimiques, mouvements...). Après une description fine enrichie de citations et de références, de nombreux exemples, de complexité variable, issus d'une diversité d'interactions, vont permettre aux enseignants de choisir les plus appropriés à leur objectif d'enseignement, chacun d'eux comprend une contextualisation et une analyse de l'extrait selon ses différentes dimensions verbales et non verbales, le signal vidéo ou audio et l'extrait de la transcription où le fait de langue est affiché en couleur et les marqueurs qui lui sont associés figurent en gras (Figure 4). Une rubrique "Pour aller plus loin" propose une liste de références bibliographiques, des explications complémentaires ou des exemples plus complexes. Ces fiches explicatives documentent les remerciements, le discours rapporté, les procédés d'atténuation, la question d'un point de vue pragmatique et les expressions de l'oral "quand même" et "trop" identifiées par les praticiens comme complexes pour l'apprenant, quel que soit son niveau de langue, en particulier dans certaines réalisations "quand même pas", "trop pas", "un peu trop"...

\footnotetext{
Fiche Explicative "Les fonctions pragmatiques du discours rapporté à l'oral"

Auteure : Carolina León Roa - Master DDL, Université Paris 8

Relectrices : Élodie Oursel - MCF Paris 8, Carole Etienne - IR Cnrs

Emplois

Le discours rapporté est employé pour citer les propos déjà formulés par un locuteur. II permet donc de rapporter, dans le présent, une parole passée. "Le discot rapporté est un outil discursif utilisé lorsque l'on veut faire référence à des propos issus d'une autre situation d'énonciation : il peut agir, selon les besoins discursifs । élément de "reportage" (dans un but narratif ou informatif) ou comme argument d'autorité (dans un but argumentatif)" (Vincent, 2004 : 237).

Le discours rapporté (DR) à l'oral est une stratégie discursive des locuteurs leur permettant de reformuler les propos d'autrui ou son propre discours (je lui ai dit c que...). Le locuteur ne se limite pas toujours à reproduire à l'identique une information, le discours peut être transformé puis intégré au propos du locuteur. "Rap signifie donc à la fois citer, c'est-à-dire reproduire intégralement un segment dit ou écrit, mais aussi résumer, reformuler, voire évoquer ou interpréter un discours" ( $\mathrm{R}$
} $2008: 3)$. On relève donc les deux formes : le discours direct et indirect.

"le DR à l'oral s'insère dans un récit d'interactions, il implique le locuteur. C'est une forme textuelle utilisée à des fins pragmatiques diverses : transmettre une nouvel rire, argumenter." (Rosier, 2008 : 24)

Le discours direct est employé lorsque le locuteur ne modifie pas le discours d'autrui : "ils m'ont dit oh non c'est nous qui vous remercions". Le discours indirect reformuler les propos du locuteur "parce qu'elles m'ont demandé si j'avais mangé de la salade", le discours indirect entraîne ainsi des changements de pronoms p! possessifs et interrogatifs.

Á l'oral, on ne se limite pas à rapporter le discours, on peut aussi rapporter, les gestes et l'intonation du locuteur. "Pour avoir un discours rapporté, il est nécessaî un discours citant et un discours cité co- ou contextuel. Si le terme discours englobe des paroles, des pensées, voire des actes de parole ou des gestes (comme un so faut que l'idée de voix (même silencieuse) reste prépondérante" (Rosier, 2008 : 21)

Dans cette fiche, nous allons présenter les fonctions pragmatiques du discours rapporté les plus fréquentes à l'oral : 
Dans cette fiche, nous allons présenter les fonctions pragmatiques du discours rapporté les plus fréquentes à l'oral :

1. Rapporter une argumentation, une explication: C'est un usage qui permet au locuteur d'argumenter ou d'expliquer ce qu'il est en train de dire. L'argumentation । locuteur de défendre ou de réfuter ce qu'il est en train de dire, et l'explication permet au locuteur de faire comprendre ses propos ou ce qui s'est passé lors d évènement.

2. Rapporter une opinion: Le locuteur peut reprendre pour la développer une opinion, une idée, un point de vue.

3. Rapporter un événement: Le locuteur peut introduire ou raconter un évènement ou une anecdote déjà passée.

4. Rapporter des critiques: Le locuteur expose des critiques formulées.

5. Rapporter un refus: Le locuteur remonte un refus pour généralement le commenter.

6. Pour aller plus loin ...

Grammaire

- Modalisateurs adverbiaux et verbaux

Minimisateurs

Lexique

Le verbe introducteur le plus utilisé dans le discours rapporté est "dire", cependant son emploi n'est pas systématique à l'oral. Le discours rapporté peut en effel par des formulations telles que "il/elle m'a dit, il/elle me dit, il nous a dit, on leur a dit, j'ai dit, $j$ 'avais dit, je lui dis...", mais aussi par la variante "je fais, il me fait" registres informels. Contrairement à l'écrit, l'emploi de la conjonction de subordination "que" n'est pas systématique.

\section{Registre}

Le discours rapporté est employé dans les registres formels ou informels., dans des activités privées comme professionnelles, telles que des réunions de travail, consultations juridiques, des visites guidées mais aussi dans des conversations entre amis, des repas...

\section{Prosodie}

- Saillance de la conjonction de subordination "que" : "les français ont dit que..", "elle m'a dit que non" et du verbe dire "alors euh quand il dit okay pour le perso "elle me dit ben soit au lycée soit chez moi", "qu'elle nous a dit" afin de marquer le début du discours rapporté.

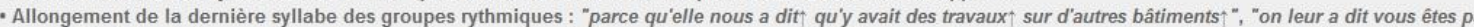
de vous réunir fin c'est quand même fou $\uparrow$ vous avez pas de projet` vous êtes formateurs vous n'avez pas de projet pour notre formation $\uparrow$ vous êtes incapables de vol - Lors de l'emploi du discours direct les locuteurs peuvent reproduire l'intonation d'un autre locuteur : intonation montante "elle dit mais vous me devez de l'arge l'argent à qui vous l'avez donné^".

- Le locuteur peut aussi employer un ton d'hésitation, "j'ai dit que ben ben si si on est gentil d'accord mais si on est trop gentil aïe aïe aïe"

\section{Marqueurs spécifiques}

Le discours rapporté peut s'accompagner de :

- marqueurs spécifiques du discours comme "hein", "bon", "ben"

marqueurs qui accompagnent l'argumentation "en fait" "donc" "parce que", "en gros", "donc en fait", "mais"

- marqueurs "après", "alors", "puis", "du coup" pour articuler plusieurs discours rapportés

- marqueurs "tu vois", "je te jure" qui ponctuent les phases de discours rapporté et sollicite les autres locuteurs

Description : Lors d'un apéritif entre amis, la locutrice (JUL) raconte la discussion qu'elle a eue avec une amie

Explication : JUL rapporte l'opinion de son amie "elle me dit euh un truc ouais mais en fait les chats ils comprennentet tout"

- Discours direct: "elle me dit euh un truc ouais ... "sans lusage de "que" - Multimodalité - Mouvement douverture des yeux pour marquer son étonnement et attirer l'attention de ses interlocuteurs sur "mais en fait les chats ils comprennent et tout"

4

Vidéo (mp4)

Transcription (docx)

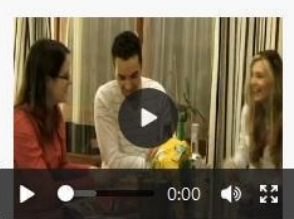

Fig. 4. Fiche "Le discours rapporté", exemple 2.2

Dans le cadre d'une unité portant sur la politesse, Carmen Alberdi a fait appel à la fiche explicative "Trop" pour sensibiliser les apprenants à différents observables et développer ainsi leurs compétences pragmatiques au-delà de la simple reconnaissance de formes lexicales, elle a sélectionné pour cela des exemples différents en fonction de leur niveau qui allait de $\mathrm{A} 2$ à $\mathrm{C} 2$ suivant ses cours. Pour présenter à ses élèves l'inter-agir dans une perspective socio-relationnelle, Elisa Ravazzolo a exposé les quatre procédés de la fiche explicative "Les procédés d'atténuation", à savoir les modalisateurs, les formules de politesse, l'explication - l'argumentation et la blague en privilégiant certains des exemples, 
puis a demandé aux étudiants dans un second temps de déterminer de quel procédé il s'agissait dans d'autres exemples de la fiche. Si le nombre important d'exemples et de rubriques peut sembler à première vue déroutant à la première lecture d'une fiche explicative, il permet néanmoins aux enseignants d'adapter son contenu à ses objectifs d'enseignement, de disposer d'une variété de situations et d'une brève analyse qui identifie les caractéristiques majeures sachant que la rubrique "Pour aller plus loin" donne la possibilité de poursuivre ses investigations.

Dans une prochaine étape, pour répondre à la demande récurrente des enseignants qui rencontrent parfois des difficultés à appréhender toutes les facettes de ces extraits d'interactions, une analyse interactionnelle des quarante extraits sera bientôt mise en ligne. Elle comprendra l'identification des actions langagières et le découpage en séquences interactionnelles, la description de l'organisation des tours de parole avec notamment les co-constructions, le rôle des chevauchements (superposition de paroles) et les prises de parole privilégiées, les fonctions des marqueurs, les marques de politesse, les dislocations et les constructions remarquables, les répétitions et les réparations.

\section{Les différents types de ressources et leur articulation}

Comme ces différents matériels pédagogiques ont été constitués et mis en ligne progressivement et que leur appropriation et leur mise en œuvre en classe de langue par les enseignants nécessite un peu de temps selon la répartition des cours dans les semestres, nous ne disposons pas encore de beaucoup d'exemples d'articulation de ces supports multimédia. Au moment de leur conception, les extraits avec leur piste d'exploitation et leur analyse interactionnelle (en cours de finalisation) avaient été pensés pour constituer une session de cours tandis que les collections venaient illustrer un point particulier rencontré par exemple dans un extrait. L'étude par exemple de séquences dans une boulangerie avec l'extrait CLAPI-FLE-2 permet d'aborder entre autres l'imparfait de politesse très utilisé dans les commerces avec "je voulais" mais qui s'avère attesté également dans un tout autre contexte notamment lors d'une invitation téléphonique entre amies dans l'extrait CLAPIFLE-9 cf la collection "L'imparfait de politesse". Les fiches explicatives par la richesse et la complémentarité de leurs contenus peuvent quant à elles alimenter une unité d'enseignement divisée en plusieurs séances.

A partir des exploitations pédagogiques dans des cours de Langue moderne en première et deuxième année de licence, Elisa Ravazzolo a montré comment elle articulait ces différentes ressources en fonction du niveau des apprenants mais également des différents objectifs : compréhension, organisation des interactions, syntaxe de l'oral et perspective interculturelle (Ravazzolo, Etienne, 2019).

Dans le cadre d'un enseignement dédié à la cuisine dans un cours de dialogue italienfrançais en première année d'interprétariat, Natacha Niemants a fait appel dans une première séance à l'extrait CLAPI-FLE-19 concernant la préparation d'un plat en utilisant le visionnage et les pistes d'exploitation proposées, puis elle a choisi dans la fiche explicative "Le discours rapporté" plusieurs exemples concernant des repas qu'elle a présentés aux étudiants dans une deuxième séance en leur demandant d'identifier le discours indirect après deux visionnages, puis de le transformer en discours direct, et ensuite de rapporter à leur tour à l'oral une anecdote de leur choix.

Voyons à présent, au sein même d'une séance de cours, comment certains corpus ont pu être proposés à des apprenants débutants et comment ils se sont intégrés à un dispositif pédagogique destiné à ce public. 


\section{Le cas particulier des apprenants débutants en FLE}

Comme nous l'avons relevé au paragraphe 2, le CECRL n'envisage pas les interactions spontanées en situation réelle pour les niveaux $\mathrm{A} 1$, très souvent confrontés aux compréhensions orales fabriquées et très simples des méthodes de langue. En effet, Le Référentiel pour le français niveau A1 (Beacco, Porquier, 2007) établit que l'apprenant A1 «peut prendre part de manière limitée aux échanges verbaux (...) mais [qu'il] n'est pas en mesure de gérer convenablement les relations sociales de base» et «sont exclues la compréhension des conversations", il ne peut pas "comprendre une interaction entre locuteurs natifs » et « ne peut ni accéder ni produire une discussion informelle ». Cependant , pour la fonction "Structurer ses propos ", l'apprenant A1 est censé pouvoir connaître certains ponctuants du discours comme "Dis donc", "bon alors", "euh", "tu sais", "bon voilà......quoi" ou "hein".

Nombreux sont les étudiants A1 qui, vivant dans un contexte homoglotte, ont accès à la langue en dehors de la classe. Si comprendre, c'est apprendre à percevoir en variant les situations (Lhote, 2001) et s'il est pertinent, pour enseigner/apprendre une langue étrangère de donner des supports d'un niveau supérieur (Courtillon, 2003), nous avons fait l'hypothèse qu'une exposition guidée des apprenants A1 à des interactions authentiques pouvait être réalisable. Nous avons voulu tester l'utilisation de CLAPI-FLE dans le cadre d'un atelier "Jeux de rôle" à destination des étudiants A1 inscrits au Service universitaire de FLE (SUFLE) de l'Université Aix-Marseille. La problématique est la suivante : est-il possible de sensibiliser des apprenants de FLE débutants à l'oral authentique ? Si oui comment alors intégrer ces corpus dans le dispositif pédagogique ? A partir de là comment didactiser ces corpus ? Et quels sont les apports des corpus d'interactions authentiques (extraits de CLAPI-FLE) par rapport aux dialogues fabriqués proposés dans des manuels de FLE récents ? Enfin cette sensibilisation concerne-t-elle plutôt la compétence de réception ou celle de production ou encore leur interdépendance?

L'expérimentation a été menée au cours de 2 séances de 1 h30 auprès de 7 apprenants du Service Universitaire de FLE, de niveau A1 (débutants et faux débutants) et de 7 nationalités différentes. L'objectif pragmatique travaillé était celui de l'invitation. La démarche a mis en perspective une invitation dans une interaction fabriquée proposée dans le manuel Compétence compréhension orale A1/A2, Clé international (2004), et une interaction d'invitation téléphonique authentique proposée dans l'extrait CLAPI-FLE-8 (http://clapi.icar.cnrs.fr/FLE/affiche_contexte.php?affiche_extrait_encours=84\&extrait_enc ours $=84$ ).

L'approche se révèle ethnographique (Cambra Giné, 2003), nous sommes à la fois l'enseignant et le chercheur, accédant ainsi au statut de praticien-chercheur (Sensevy, 1995) à travers une démarche de "participation observante » (Blanchet et Chardenet, 2011). Deux types de recueil de données complètent le dispositif de classe. Des captations vidéo des deux séances ont été réalisées par une personne extérieure placée au fond de la classe de telle sorte qu'elle puisse capter les interactions entre l'enseignante et les apprenants. À ces captations s'ajoutent des productions écrites d'apprenants dont la tâche consistait à transformer l'interaction fabriquée de départ afin de la rendre plus spontanée.

L'exploitation pédagogique se décompose en quatre temps:

Etape 1 : une première exploitation de la compréhension orale autour de l'interaction fabriquée niveau A1 qui débouche sur la découverte des actes de parole propres à 
l'invitation, accompagnée de la transcription du document sonore. On souligne l'absence d'authenticité des échanges, sans aucune ouverture ni clôture de l'interaction, sans aucun ponctuant du discours.

F1- Tu veux faire du vélo avec nous dimanche matin?

F2- Oui, c'est une bonne idée. Vous allez où ?

F1- Au bord de la mer, c'est facile. Tu peux venir avec ta fille.

F2- Pourquoi pas, elle adore faire du vélo

F1- Mes enfants aussi, ils aiment beaucoup ça

F2- Et à midi, on fait un pique-nique ?

F1- Non, on mange à la maison, je vous invite !

F2- Ah merci, c'est gentil

Fig. 5. Transcription de l'interaction fabriquée

(Compétence orale, A1/A2, 2004, Clé)

Etape 2 : une deuxième activité de compréhension orale globale de l'interaction de l'invitation téléphonique proposée par CLAPI-FLE. Cette dernière est ensuite accompagnée de la transcription que nous avons dû simplifier afin de la rendre plus lisible (remplacement $\mathrm{du}$ code de transcription par une ponctuation; annulation du marquage temporel; simplification de certains termes, mise en valeur des marques de l'oral). Si CLAPI-FLE propose par ailleurs une transcription simplifiée, celle-ci n'intègre pas la ponctuation connue par les apprenants, que nous avons dû rétablir pour leur faciliter la lecture. 


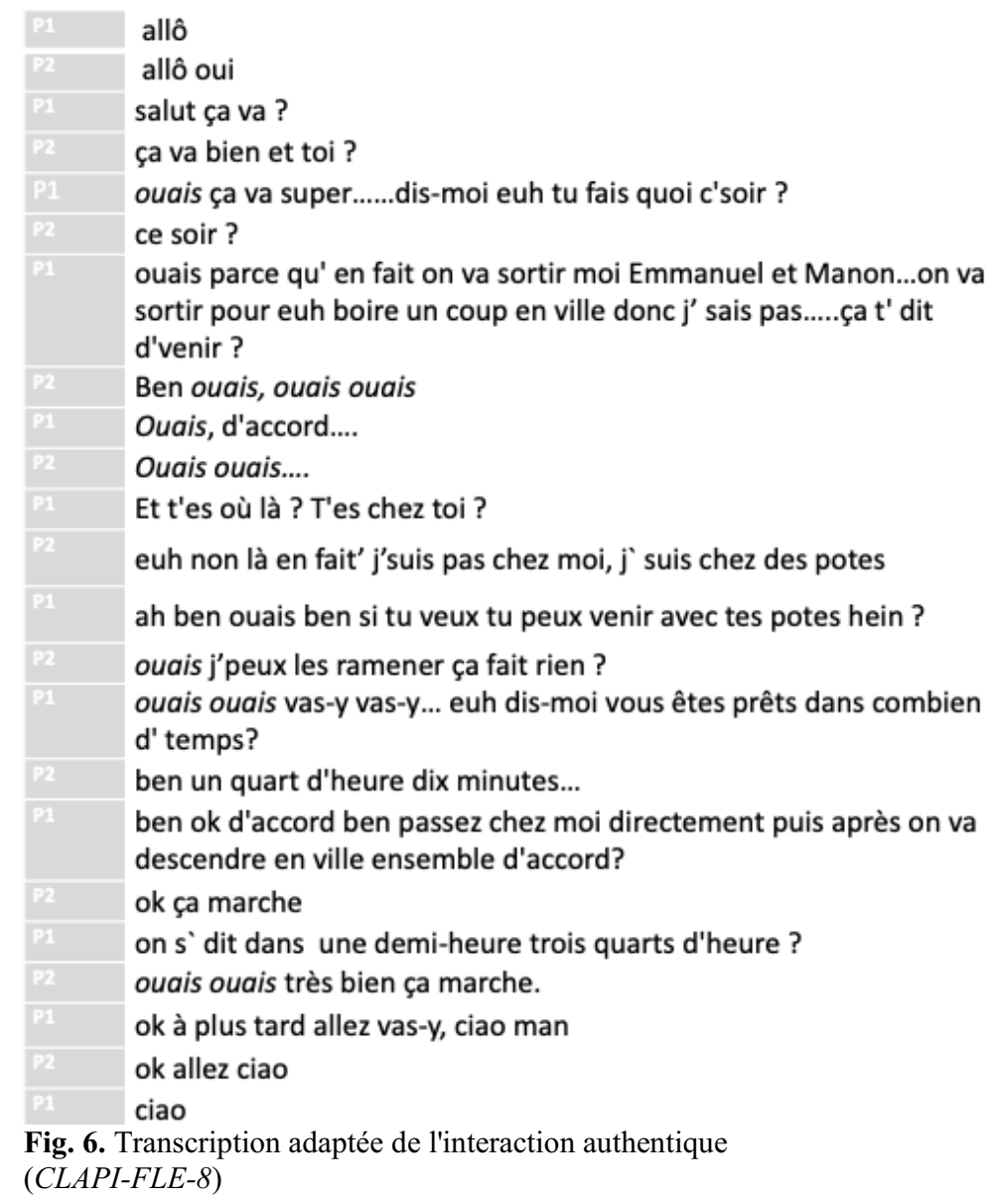

Etape 3: une approche inductive des marques de l'oralité présentes dans l'interaction de CLAPI-FLE. Les étudiants sont invités à lire le dialogue par deux, ils prennent conscience que la lecture est difficile parce que la syntaxe des phrases n'est pas habituelle. On leur demande ensuite de relever les marques de l'oral spontané. Même si leur formulation n'est pas aisée, on peut dire, avec André (2018 : 12) « qu' ils observent, comparent, analysent le fonctionnement de la langue en cotexte et en contexte. Ils [essaient de déduire] eux-mêmes les règles, les usages et les variations sociolinguistiques des pratiques langagières $\gg$. Les apprenants relèvent la présence d'une ouverture et d'une clôture, des mots et expressions familières, des ponctuants du discours (euh, ben, en fait), des formes tronquées, des répétitions, la réitération de "ouais". La transposition didactique (André, 2018) des recherches sur les interactions en classe de FLE, surtout si le public est de niveau A1, nécessite une véritable adaptation du discours de l'enseignement au niveau métalinguistique. Les captations vidéo révèlent des apprenants A1 attentifs et participatifs mais qui ont beaucoup de difficultés pour exprimer ce qu'ils ressentent. Ce qui confère à l'enseignant une présence très forte, à la fois comme accompagnateur et comme vecteur d'informations, une constatation qui rejoint V. André $(2018: 16)$ pour qui :

« L'enseignant peut confirmer ou infirmer ces hypothèses, il peut en suggérer d'autres, expliquer le fonctionnement repéré, aider à l'analyser, mettre sur la voie, accompagner les démarches, etc. En procédant de cette façon, les apprenants, accompagnés 
d'enseignants formés aux liens entre sociolinguistique et didactique, acquièrent des compétences sociolangagières et apprennent à interagir de façon appropriée »

En visualisant les interventions des apprenants, on constate aussi une plus forte participation des apprenants faux-débutants habitant en France depuis plus longtemps. L'ensemble des débutants, quant à eux, qui vivent en milieu homoglotte, manifestent un vif intérêt pour ce type de ressources. Ils reconnaissent certains petits mots et expressions de l'oral de leur quotidien qui jusqu'à présent perturbaient leur compréhension globale mais qu'ils identifiaient comme utiles par leur fréquence, par exemple "ouais", "en fait", "ben", "tu fais quoi ?"

Etape 4: la transformation, à l'écrit, par les apprenants rassemblés en petits groupes, de l'interaction fabriquée en dialogue plus naturel.

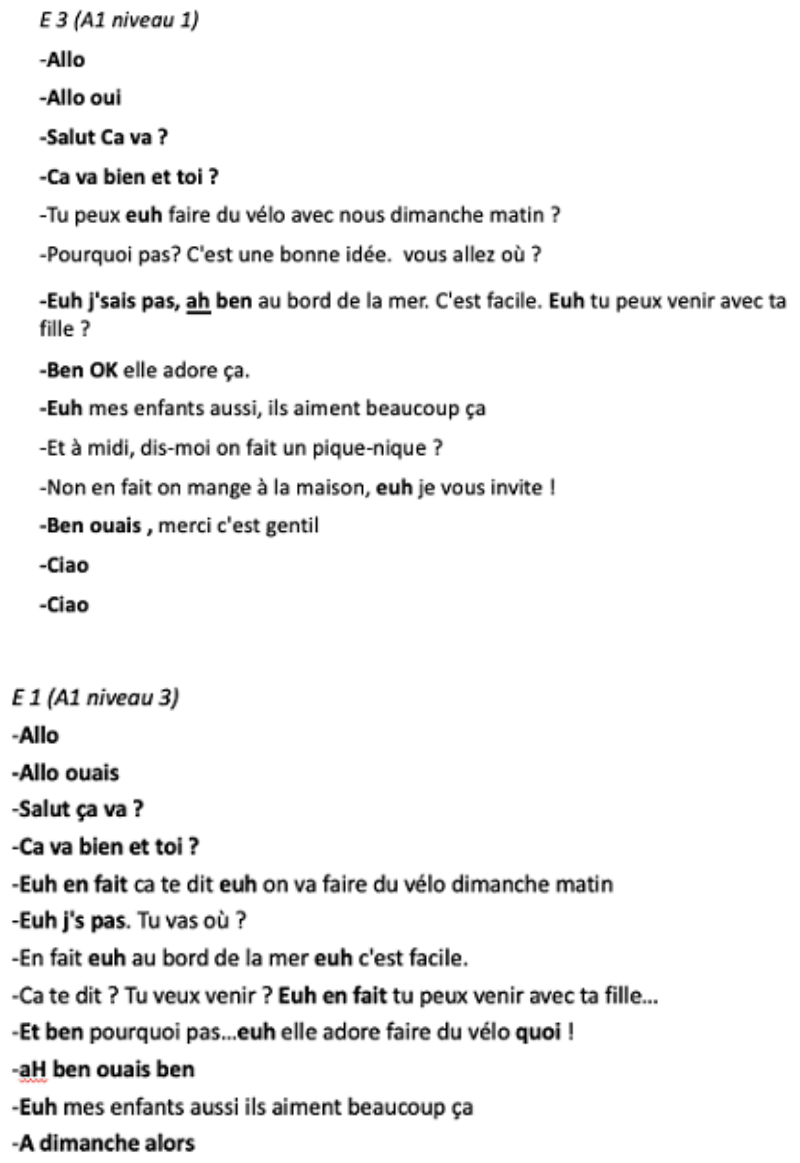

Fig. 7. Productions écrites d'apprenants A1

Les productions révèlent que les apprenants ont été sensibles à certaines marques de l'oral, comme la nécessité d'ouvrir et de fermer la conversation, ainsi qu'à l'utilisation de certains termes comme "euh", "en fait", "ben", "quoi". Nous faisons l'hypothèse que ces termes sont des marques qu'ils entendent en dehors de la classe. On constate tout de même qu'elles ne sont pas toujours placées de manière cohérente dans l'interaction, l'incohérence 
étant plus évidente dans le dialogue écrit par des apprenants débutants de niveau 1. Ces copies rendent compte de l'appropriation en cours, même au niveau A1, d'un français parlé qu'ils peuvent entendre à l'extérieur du contexte guidé de la classe.

Cette expérimentation nous conforte dans l'idée qu'il est possible et stimulant de confronter des apprenants A1 à un oral naturel en cours de FLE, à condition que la démarche soit guidée. La question qui demeure est celle de savoir si cette activité permet de travailler la réception et/ou la production en termes de performances. Car le fait d'écrire des marques de l'oral dans un exercice, ne signifie pas pour autant que l'apprenant sera capable de les produire à l'oral en situation réelle, nous faisons l'hypothèse que ces dernières s'acquièrent peut-être plus par imprégnation avec le milieu écologique, du moins au stade des étudiants primo-arrivants. En tant qu'enseignante de FLE, cette expérimentation nous a permis de faire évoluer nos pratiques : nous n'hésitons plus à donner, aux apprenants $\mathrm{A} 1 / \mathrm{A} 2$, des interactions authentiques pour compléter leur manuel de cours, à condition de bien guider l'exploitation. En tant que chercheure, et nous intéressant à la problématique des classes multi-niveaux en FLE, cette expérimentation permet de penser le choix et la manière d'exploiter un support authentique pour travailler avec des apprenants de niveaux différents, intégrant des apprenants A1 dans une exploitation en classe entière. Le site CLAPI-FLE peut se prêter à des exploitations multi-niveaux d'un nombre significatif de documents audio-visuels.

Par la suite, nous avons travaillé avec la même exploitation de CLAPI-FLE sur l'invitation avec des apprenants $\mathrm{B} 2+/ \mathrm{C} 1$ à la fin d'une séance, toujours pour faire prendre conscience des marques de l'oralité. Les étudiants ont réalisé que ce n'est pas tant la difficulté du lexique qui rend un dialogue authentique difficile à comprendre mais plutôt sa structure, " son architecture complexe d'emboîtements successifs » (Alberdi, 2007) qui est au centre de la compétence interactionnelle, peu travaillée en classe de FLE en France et dans les centres de FLE à l'étranger (Ravazzolo \& al, 2015). Les apprenants de niveau avancé découvrent ce dispositif avec leur enseignant et sont encouragés à travailler en autonomie en exploitant ces ressources multimodales.

Les ressources CLAPI ont été proposées à des étudiants $\mathrm{B} 1$ au cours de la période de confinement en mars-avril 2020, afin qu'ils continuent, en autonomie, à se confronter à des interactions authentiques. Certains ont mentionné la difficulté à utiliser la plateforme par eux-mêmes même s'ils ont trouvé très pertinente l'écoute de certains échanges accompagnés des transcriptions. Ces retours vont dans le sens d'une réflexion sur le guidage de l'enseignant.

\section{Perspectives}

Si cet article a retracé la collaboration depuis bientôt une dizaine d'années entre les linguistes spécialistes de l'interaction du laboratoire ICAR et le réseau de praticiens didacticiens et enseignants pour construire ensemble des ressources pour l'enseignement du français oral, et plus particulièrement de la compétence d'interaction, il a suggéré plusieurs pistes d'exploitation dès le niveau débutant de ces ressources qui peuvent paraître difficiles au début de l'apprentissage.

Cette approche défend que l'exposition dès les premiers contacts à une variété de réalisations du français parlé en interaction permet d'appréhender cette diversité, de comprendre les procédés verbaux et non verbaux mis en place par les participants au cours de son déroulement, dans l'objectif de mieux les comprendre et de les reproduire par la suite dans des contextes similaires où ils font sens, que ce soit dans une forme approchée ou dans une organisation voisine articulant les mêmes mécanismes. 
En parallèle de CLAPI-FLE, nous venons de finaliser le projet CORAIL (http://clapi.icar.cnrs.fr/Corail) utilisable directement par l'apprenant pour découvrir le français oral dans différents contextes, comprendre les expressions figées les plus fréquentes et apprendre à réaliser les principaux actes de communication indispensables pour apprendre à interagir, mais difficiles à assimiler et à analyser dans la dynamique des échanges, en particulier quand ils soulèvent des questionnements liés à des différences interculturelles. Dans cette perspective, le projet CORAIL pourra être présenté à différents publics d'apprenants enfants, étudiants ou adultes suite à des migrations économiques, politiques ou environnementales, qui doivent faire face au quotidien à des situations d'interactions similaires sans en maitriser les rouages au détriment de leur insertion sociale, scolaire ou professionnelle.

\section{Références bibliographiques}

Alberdi, C. (2007). Politesse et Structure de l'interaction, Pragmalinguistica, 15-16, 7-32

Alberdi, C., Etienne, C., Jouin-Chardon, E.,(2018). Les apports des corpus d'interactions naturelles en situation de classe: enjeux et pratiques, Action Didactique 1, 55-70

André, V. (2018). Nouvelles actions didactiques : faire de la sociolinguistique de corpus pour enseigner et apprendre à interagir en français langue étrangère, Action Didactique 1, 71-88

Beacco, J-C., Porquier, R. (2007). Les Référentiels, niveau A1, Conseil de l'Europe.

Blanchet, P., Chardenet, P. (2011). Guide pour la recherche en didactique des langues et des cultures : approche contextualisée, Paris : Broché

Cambra Giné (2003). Une approche ethnographique de la classe de langue, Paris : Didier

CECRL, (2018). Cadre européen commun de référence pour les langues: apprendre, enseigner, évaluer, Volume complémentaire avec de nouveaux descripteurs, Paris : Didier

Courtillon, J (2003). Elaborer un cours de FLE, Paris : Hachette.

David, C. Abry, D. (2018). Classes multi-niveaux et pédagogie différenciée. Hachette

David, C.(2015). Pratique enseignante dans des classes multilingues et multi-niveaux en FLE: une gestion complexe ces supports / outils. Français dans le monde. Recherches et applications, 2015, La grammaire en FLE/FLS: quels savoirs pour quels enseignements? 57, 136-148

Détienne, Françoise/Traverso, Véronique (2009). Méthodologies d'analyse de situations coopératives de conception: Corpus MOSAIC, Nancy, Presses universitaires de Nancy.

Etienne, C., Jouin, E., (2019). Constituer des ressources pédagogiques pour enseigner le français oral à partir des recherches menées en interaction. Peter Lang. Variation, plurilinguisme et évaluation en FLE, Collection Sprache, Mehrsprachigkeit und sozialer Wandel. 225-240

Goodwin, C. (2000). Action and embodiment within situated human interaction. In : Journal of Pragmatics 32, 1489-1522.

Groupe ICOR (Baldauf-Quilliatre, H., Colon de Carvajal, I., Etienne, C., Jouin-Chardon, E., TestonBonnard, S., Traverso, V.) (2016). CLAPI, une base de données multimodale pour la parole en interaction : apports et dilemmes, Avanzi M., Béguelin M.-J. \& Diémoz F. (eds), Corpus de français parlés et français parlés des corpus, Cahiers Corpus.

Holec, H. (1990). Des documents authentiques, pour quoi faire. Mélanges Crapel 20, 65-74.

Kerbrat-Orecchioni, C. (1990-1994). Les Interactions verbales. Paris : Colin, 3 vols.

Kerbrat-Orecchioni, C. (2001). Les actes de langage dans le discours : Théories et fonctionnement, Nathan

Kerbrat-Orecchioni, C. (2002). Système linguistique et ethos communicatif. Cahiers de praxématique 38. Montpellier : Pulm. 35-57

Kerbrat-Orecchioni, C.,Traverso, V. (2008). Les interactions en site commercial : Invariants et variations, Lyon, ENS Editions.

Lhote, E. (2001). Enseigner l'oral en interaction. Percevoir, écouter, comprendre, Paris: Hachette

Mondada L. (2007). Multimodal resources for turn-taking : Pointing and the emergence of possible next speakers,Discourse Studies 9/2 : 195-226. 
Mondada L. (2006). Video Recording as the Reflexive Preservation and Configuration of Phenomenal Features for Analysis, H. Knoblauch, J. Raab, H.-G. Soeffner \& B. Schnettler (éd.) Video-Analysis Methodology and Methods, Qualitative Audiovisual Data Analysis in Sociology.Oxford : Peter Lang, 51-68.

Piccoli, V., Ticca, A. C. \& Traverso, V. (2019). « Go internet it's here » : démarches administratives de personnes précaires ou en demande d'asile, Langage et société 167, 81-110.

Potter, Jonathan (2006). Naturalistic Data, Victor Jupp (ed.), The Sage Dictionary of Social Research Methods, London:Sage. Brockington.

Ravazzolo, E., Etienne, C. (2019). Nouvelles ressources pour le FLE à partir des études en interaction. $\operatorname{LINX} 79$, numéro 2.

Ravazzolo, E., Traverso, V., Jouin-Chardon, E., Vigner, G. (2015). Interactions,dialogues, conversations : l'oral en français langue étrangère. Paris : Hachette, coll. FLE

Sacks, H. (1992). Lectures on conversation. Oxford : Blackwell, 2 voll.

Sacks, H., Schegloff, E.A. \& Jefferson, G. (1974). A Simplest Systematics for the Organization of Turn Taking for Conversation, Language 50(4), 696-735.

Sensevy, R. (1995). Le sens du savoir, Eléments pour une théorie de l'action conjointe en didactique, Louvain : De Boeck

Thomas, A., Granfeldt, J., Jouin-Chardon, E. \& Etienne, C.(2016) Conversations authentiques et CECR : compréhension globale d'interactions naturelles par des apprenants de FLE. Cahiers de l'AFLS 20:2, $1-44$

Traverso, V (1998). La conversation familière, Lyon, Presses universitaires de Lyon, L'Harmattan.

Traverso, V. (2011). Chevauchements de parole, résolution et réparation dans la conversation en ligne, Develotte, Christine/Kern, Rick/Lamy, Marie-Noëlle (éds.), Décrire la conversation en ligne, Le face à face distanciel, Lyon, ENS Éditions, 117-145.

Traverso, V. (2016). Décrire le français parlé en interaction, Paris, Ophrys.

Traverso, V. (2017). Évaluations et compliments : de la visite amicale à la visite touristique, Recherches en linguistique et en didactique : apports de l'analyse des corpus et des interactions. Editions Lambert-Lucas, 181-206. 